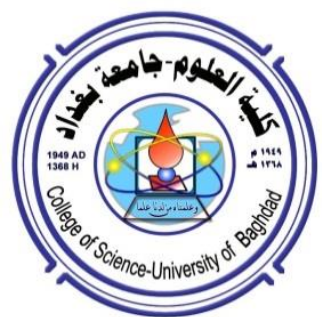

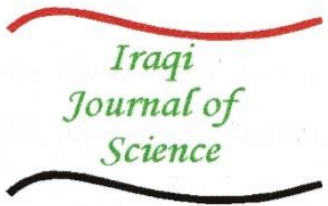

ISSN: 0067-2904

\title{
Modelling the Role of the Inner Electrode Radius on the Oxygen Negative Corona Discharge in Coaxial Electrode Geometry
}

\author{
Muayad Abdullah Ahmed \\ Department of Physics, College of Education for Pure Science, University of Mosul, Mosul, Iraq
}

Received: $23 / 4 / 2021$

Accepted: 17/6/2021

\begin{abstract}
The charge species plays a vital role in changing the field in direct current discharge (DC). This article introduces a numerical modeling in one dimension of the inner electrode diameter of oxygen-fed negative corona discharge in coaxial electrodes geometry. The properties of negative corona plasma in a concentric cylindrical electrodes (wire-cylinder) were simulated by COMSOL Multiphysics software. Various diameters of negative corona electrode, namely $0.01,0.025,0.05$, 0.075 , and $0.125 \mathrm{~mm}$, were applied, where the diameter of the outer cylindrical electrode was taken as $15 \mathrm{~mm}$. The model was run at atmospheric pressure and the applied negative voltage was $-10 \mathrm{KV}$. Moreover, oxygen gas was used to fill the inter-electrodes distance. Furthermore, the spatial distribution of electrons, positive ions, and negative ions as a function of the diameter of negative electrode of the negative corona discharge were investigated. The study also tested the effects of the electrode diameter of the negative corona discharge on ozone generation. The observed decrease in ozone density with the increase in negative electrode diameter was reasonable and consistent with other results provided by researchers in this field.
\end{abstract}

Keywords: Negative corona discharge, ozone generation, COMSOL Multiphysics,

\section{نمذجة دور القطب الداخلي على تفريغ الهالة السالبة في الاوكسجين في منظومة الاقطاب المتحدة المركز$$
\text { مؤيد عبدالله أحمد }
$$ \\ قسم الفيزياء، كلية التربية للعلوم الصرفة، جامعة الموصل، الموصل، العراق احلاق}

$$
\begin{aligned}
& \text { الخلاصة } \\
& \text { تلعب أنواع الثحنات الكهربائية دورًا مهمًا في تغيير المجال في التفريغ التيار المستمر. تعاملت هذه }
\end{aligned}
$$

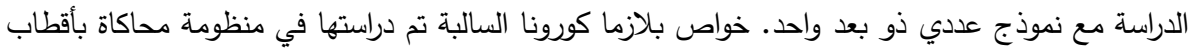

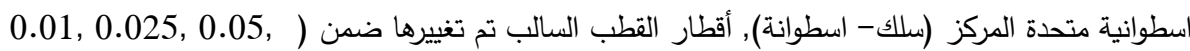

$$
\begin{aligned}
& \text { 1 0.075, } 0.125 \text { mm }
\end{aligned}
$$

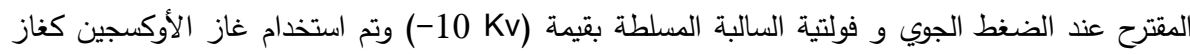

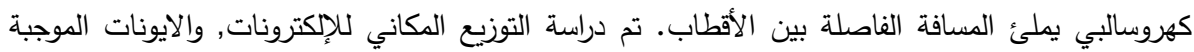

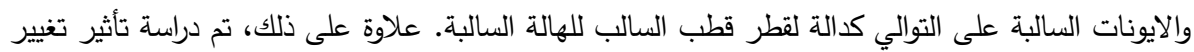

*Email: moyadalharbi@uomosul.edu.iq 


$$
\begin{aligned}
& \text { القطب السالب للهالة على انتاج الاوزون. اظهرت النتائج انخفاض كثافة الاوزون مع القطب السالب للهالة } \\
& \text { بنسب مقبولة متفقة مع نتائج لدراسات سابقة في هذا المجال. }
\end{aligned}
$$

\section{Introduction}

Electrical corona discharge is considered as a type of self-sustained discharges that appears around the pin and along metals. Despite the widespread applications of corona discharge, there is no perfect simulation model for electrical discharge that shows high accuracy. Continuous or pulsed electrical discharge is possible at atmospheric pressure, where the shape of the discharge depends on the high voltage source applied [1]. The high accuracy depends on the characteristics of the I_V and corona discharge, including electric current density and plasma density. Most of the previous attempts focused on this discharge [2]. For example, Abdel-Salam [3] used a new finite element to solve the second order partial differential equation (PDE). The effectiveness of this method was tested with several geometrical shapes and a good precision was obtained using the computer programing.

In fact, the direct current corona discharge can be divided into two types; the positive and the negative corona discharge. This distinction depends on the connection between the source polarity and the corona discharge electrodes. Cross and Beattie [4] studied the positive corona discharge in a mixture of oxygen and nitrogen gases in quasi electric fields. Both the electrical and optical characteristics were obtained. More studies were conducted to measure and compute the surface field in DC corona discharge by solving Passion's equation [5, 6]. Khalifa and Abdel-Salam [5] calculated the surface field and indicated that the avalanche in negative DC discharge developed in the discharge region. This region is consisted of two layers; the inner layer which is generally made of positive ions and the outer layer which is mostly made of negative ions.

Typically, the positive terminal of high voltage (HV) power supply is connected with thin electrode diameter in the positive corona discharge. Whereas, the negative polarity for $\mathrm{HV}$ goes to the large diameter electrode for the asymmetry discharge system which represents the grounded electrode. Likewise, in the situation of coaxial wire-cylinder geometry, the inner electrode is a small diameter wire [7]. Conversely, at a high voltage polarity, the HV positive terminal goes to the cylinder or the outer large diameter of electrode. In this case, a negative corona discharge is created $[8,9]$. In fact, the competition between the two types of direct current corona discharges is an important matter. It allows to distinguish the properties of corona discharges and demonstrate the similarities and differences between them, by using several geometric shapes of the reactor electrodes [10,11]. Measurements and numerical analysis received remarkable attention for defining $\mathrm{I}-\mathrm{V}$ characteristics. Zheng et al. [12] studied the I-V characteristics of DC corona discharges between coaxial cylinders in air. Mustafa et al. [13] used an infinite element method by using COMSOl Multiphysics software in concentric cylindrical electrodes. They tested the effects of temperature on the current and voltage characteristics of the negative corona discharge in air. They found a good agreement between the theoretical and experimental results. The ozone generation in negative corona discharge is larger than that in positive corona discharge. Most numerical and experimental studies dealt with the effects of a number of parameters on ozone generation at atmospheric pressure in dry air or in oxygen gas that represents an electro-negative gas. It has the ability to accept electrons and create negative ions or a mixture of nitrogen and oxygen gases in different geometric systems, such as the coaxial wire-cylinder and wire- plain. Ozone generation is affected by the inner wire diameter, the distance between the two electrodes, and the electron temperature. In addition, the effects of changing the diameter of the inner electrode in different species of negative corona discharge were extensively investigated [1419].

The main aim of the present study is to analyze the effects of the presence of DC negative corona discharge on the activity of oxygen gas $\left(\mathrm{O}_{2}\right)$, which represents an electronegative gas. 
The study introduces a numerical model by using commercial COMSOl Multiphysics software. The effects of the temperature on ozone generation, as well as the effects of electrons, positive ions, and negative ions distribution, were also investigated.

\section{Methodology}

\section{The model}

The model provides a simulation of direct current of corona electrical discharge using a system of coaxial electrode geometry (wire-cylinder) in one dimension. The corona discharge is a self-sustained discharge in atmospheric pressure of oxygen gas. This axial symmetry system deals with different charged particles; i.e. electron, positive ions, and negative ions.

The design of the coaxial system is the factor that is responsible of generating the corona discharge in this study. When the negative potential difference reaches a certain voltage value across the inner electrode, the inter-electrode distance divides in two zones one of them is thin layer around the axial wire, called the ionization region, while the second part is called the drift region. The most important interactions occur in the ionization region [20]. The limited capability of our computer imposed us to use a one dimensional geometrical shape in this analysies. The used axial geometry shape is composed of a small diameter wire, that represents corona electrode, and a cylinder that represents the grounded electrode. The cylinder electrode is $15 \mathrm{~mm}$ in diameter but the inner electrode wire has different diameters $(0.01,0.025,0.05,0.075,0.125 \mathrm{~mm})$. The discharge conditions involved oxygen gas at atmospheric pressure with a fixed $\mathrm{HV}$ of $10 \mathrm{KV}$. This geometry enabled us to decrease time and effort during the simulation procedure. Besides, changing the inner electrode diameter.

Corona discharge is a hydrodynamic model that consists of drift and diffusion, as expressed in the equation given below:

$$
\frac{\partial \mathrm{N}}{\partial t}+\nabla \cdot \Gamma=S
$$

$\mathrm{N}$ represents the particle density, $\Gamma$ represents the flux density, and $S$ is the source. The concept presented in equation (1) contains the creation (production) or destruction of particles [20]. $\Gamma$ can be written as:

$$
\Gamma=\mu E-D \nabla N
$$

The drift flux in equation (2) is described by the mobility of the particle and depends on the field. The second term is the gradient of the density $N$ (species type), while $D$ represents the diffusion coefficient [21]. The diffusion of the ion and the electron can be expressed according to Einstein relation as [22]:

$$
D=\frac{\mu K T}{|q|}
$$

where $\mu, K, T, q$ are the mobility, Boltzmann constant, temperature, and charge particle, respectively.

The continuity equations of the positive charged particles, negative ions, and electrons in the cylindrical coordinates are written as $[11,14,23]$ :

$$
\begin{gathered}
\frac{\partial N_{e}}{\partial t}+\frac{1}{r} \frac{\partial\left(r N_{e} V_{e}\right)}{\partial r}-D_{e} \frac{1}{r} \frac{\partial}{\partial r}\left(r \frac{\partial N_{e}}{\partial r}\right)=(\alpha-\eta) N_{e} V_{e}-\beta_{e+} N_{e} N_{+} \\
\frac{\partial N_{+}}{\partial t}+\frac{1}{r} \frac{\partial\left(r N_{+} V_{+}\right)}{\partial r}=\alpha N_{e} V_{e}-\beta_{e+} N_{e} N_{+}-\beta_{-+} N_{-} V_{-} N_{+} \\
\frac{\partial N_{-}}{\partial t}+\frac{1}{r} \frac{\partial\left(r N_{-}\right)}{\partial r}=\eta N_{e} V_{e}-\beta_{-+} N_{-} N_{+} \\
\nabla \cdot\left(-\varepsilon_{0} \nabla V\right)=e\left(N_{+}-N_{e}-N_{-}\right)
\end{gathered}
$$


The subscripts in equations $(5,6,7)$ relate to the particles produced in oxygen negative corona plasma, where e, + , - refer to the electrons, positive, and negative ions, respectively. The symbols $N_{-}, N_{e}, N_{+}$denote densities of the negative, positive ions, and electrons, respectively. $V_{-}, V_{+}, V_{e}$ represent drift velocity values of negative, positive ions, and electrons, respectively. The symbols $\alpha, \beta, \eta$ and $D$ denote to ionization, attachment recombination, and diffusion coefficient, respectively, while $\varepsilon_{0}$ is the electric permittivity. By taking the fluxes of the particles into account, the continuity equation for each particle can be described as [21]:

$\frac{\partial N_{e}}{\partial t}-\nabla \cdot\left(N_{e} \mu_{e} E+D_{e} \nabla N_{e}\right)=S_{e}$

$\frac{\partial N_{+}}{\partial t}-\nabla \cdot\left(N_{+} \mu_{+} E+D_{+} \nabla N_{+}\right)=S_{+}$

$\frac{\partial N_{-}}{\partial t}-\nabla \cdot\left(N_{-} \mu_{-} E+D_{-} \nabla N_{-}\right)=S_{-}$

where $\mu E$ represents the drift velocity, $S_{e}$ denotes to the electrons, and $S_{+}$and $S_{-}$refer to the positive and negative ions, respectively. Since the field between the electrodes is experimentally found from the deformation in the charge space, These charges usually collect between the two

electrodes, it can be calculated by solving the Poisson equation:

$\nabla .(\varepsilon \nabla \varphi)=-q\left(N_{+}-N_{e}-N_{-}\right)$

where $\varepsilon$ is the absolute permittivity of air, which can be calculated as follows:

$\vec{E}=-\vec{\nabla}$

\section{Boundary conditions}

The boundary conditions represent the essential part that contributes in the simulation of the model. The boundary conditions of the flux are ineluctable for all the particles at the two electrodes [16]. In this model the wire-cylinder configuration contains two boundary conditions. The first is the external surface of wire electrode which is the cathode, whereas the second condition denotes that the bottom surface of the cylinder represents the grounded electrode. Now, the boundary conditions can be classified as: 1) the voltage applied on the inner electrode depends upon the voltage drop in the external circuit, the current value is low, and the magnitude of the voltage at the grounded electrode is equal to zero. However, the negative and positive ions depend on the systematic of continuity equation; therefore, the $N_{-}$ and $N_{+}$are set to zero on the two electrodes. 2) the lost electrons to the wall are attributed to the random motion, which depends on the thermal velocity and electrons energy. Simultaneously, the secondary electrons are emitted from the cathode surface as a result of ions impinging. These electrons are proportional to the secondary ionization coefficient. The below equation describes this proportionality [24]:

$$
N_{\text {secondary }}=\gamma N_{p c} \frac{\mu_{+}}{\mu_{e}}
$$

where $N_{p c}, N_{\text {secondary }}$, and $\mu_{+} / \mu_{e}$ denote positive ions, electrons density at the electrode, and mobility ratio (ion to electron mobility), respectively. The secondary ionization coefficient $\gamma$ value is in the order $10^{-2}$ [8].

\section{Mesh and numerical calculation}

The accuracy can be determined based on any method that depends on the numerical analysis, such as the finite element analysis which is based on the meshes used. These meshes usually divide the model into infinitesimal elements. This leads to solve the special physical model with more accuracy at each node of mesh elements. Providing smaller and smaller elements is required to obtain a sufficient meshing, then approaching almost to the real solution. Generally, these elements are called the degree of freedom (DOF). The DOF is related to the number of nodes and arithmetic points which determine the shape of each specific element. The computational limitations need to solve the DOF model, which are directly related to the finite element method. The increasing number of meshes brings some disadvantages, like time 
and effort consumption and high computer speeds to offset the mathematical operations. A symmetry scale was used to reduce the size of the model. It is possible to define several measures to improve the mesh. The one dimensional model can be divided to a number of separated points (field elements) distributed according to the arithmetic sequence along the line that represents the inter-electrode distance. The number of these points depends on the precession required for the simulation results. The commercial COMSOL Multiphysics software version 5.3. was used for modeling.

Twenty-nine reactions were applied, eleven of them were included in the elementary model of negative corona discharge in continuous electrostatic discharge of oxygen gas. These interactions and the ionic species represent the basic structure of the model. A large number of reactions and particles can be included in this model. However, due to the limited memory, limited central processor, and the need for long processing time, the number and the types of reactions that can be used in this model are restricted.

This model includes one elastic collision and thirteen excitation reactions, as shown in Table 1. The elastic collision and excitation reactions are used only in the force balance equation, whereas they are not considered in the mass balance equations [25]. In the elastic collision, the momentum is redistributed between the colliding particles and the total kinetic energy remains constant. The reactions shown in table 1 include two attached reactions, thirteen excitation reactions, three dis excitation reactions, five recombination reactions, one ionic reaction, two association reactions, and two ion exchange reactions. The collision does not undergo a change in the path of the incident particles only, but also leads to an increase in the diffusion time of the plasma. The existent reaction in this model is a single attachment reaction ( electron attach) where the main and basic process is that of losing an electron to produce negative ions . basically the negative ions represent negative oxygen ions $\left(\mathrm{O}^{-}\right)$, effectively during EN electrical breakdown, as shown in Table 1.

Table1- A list of reactions that used in the simulation of the negative corona discharge. The unit: $m^{3} \cdot s^{-1}$ expresses two body reactions and the unit: $m^{6} \cdot s^{-1}$ expresses three body reactions. Gas temperature is in expressed in Kelvin.

\begin{tabular}{|c|c|c|c|c|}
\hline No. & Reactions & Type & $\begin{array}{l}\text { Reaction rate constant } \\
\mathrm{m}^{3} \cdot \mathrm{s}^{-1} \text { or } \mathrm{m}^{6} \cdot \mathrm{s}^{-1}\end{array}$ & Reference \\
\hline $\mathrm{R}_{1}$ & $e+O_{2}=>e+O_{2}$ & Elastic scatt. & $f(E / N)$ & [26] \\
\hline $\mathrm{R}_{2}$ & $e+O_{2}=>O+O^{-}$ & Attachment & $f(E / N)$ & [26] \\
\hline $\mathrm{R}_{3}$ & $e+O_{2}=>O_{2}^{-}$ & Attachment & $2 \times 10^{-41} 300 / T_{c}$ & [27] \\
\hline $\mathrm{R}_{4}$ & $e+O_{2}=>e+O_{2}$ & Excitation & $f(E / N)$ & [26] \\
\hline $\mathrm{R}_{5}$ & $e+O_{2}=>e+O_{2}$ & Excitation & $f(E / N)$ & [26] \\
\hline $\mathrm{R}_{6}$ & $e+O_{2}=>e+O_{2}$ & Excitation & $f(E / N)$ & [26] \\
\hline $\mathrm{R}_{7}$ & $e+O_{2}=>e+O_{2}$ & Excitation & $f(E / N)$ & [26] \\
\hline $\mathrm{R}_{8}$ & $e+O_{2}=>e+O_{2}$ & Excitation & $f(E / N)$ & [26] \\
\hline $\mathrm{R}_{9}$ & $e+O_{2}=>e+O_{2}$ & Excitation & $f(E / N)$ & [26] \\
\hline $\mathrm{R}_{10}$ & $e+O_{2}=>e+O_{2}$ & Excitation & $f(E / N)$ & [26] \\
\hline $\mathrm{R}_{11}$ & $e+O_{2}=>e+O_{2} a_{1} d$ & Excitation & $f(E / N)$ & [26] \\
\hline $\mathrm{R}_{12}$ & $e+O_{2} a_{1} d=>e+O_{2}$ & dis Excitation & $f(E / N)$ & [26] \\
\hline $\mathrm{R}_{13}$ & $e+O_{2}=>e+O_{2} b_{1} s$ & Excitation & $f(E / N)$ & [26] \\
\hline $\mathrm{R}_{14}$ & $e+O_{2} b_{1} s=>e+O_{2}$ & dis Excitation & $f(E / N)$ & [26] \\
\hline $\mathrm{R}_{15}$ & $e+O_{2}=>e+O_{2} 4.5$ & Excitation & $f(E / N)$ & [26] \\
\hline $\mathrm{R}_{16}$ & $e+O_{2} 4.5=>e+O_{2}$ & dis Excitation & $f(E / N)$ & [26] \\
\hline $\mathrm{R}_{17}$ & $e+O_{2}=>e+O+O$ & Excitation & $f(E / N)$ & [26] \\
\hline $\mathrm{R}_{18}$ & $e+O_{2}=>e+O+O_{1} s$ & Excitation & $f(E / N)$ & [26] \\
\hline $\mathrm{R}_{19}$ & $e+O_{2}=>e+O+O_{1} s$ & Excitation & $f(E / N)$ & [26] \\
\hline $\mathrm{R}_{20}$ & $e+O_{2}=>2 e+O_{2}^{+}$ & ionization & $f(E / N)$ & [26] \\
\hline $\mathrm{R}_{21}$ & $O^{-}+O_{2}=>O_{3}+e$ & Recombination & $3 \times 10^{-16}$ & [28] \\
\hline
\end{tabular}




\begin{tabular}{lcccc}
\hline $\mathrm{R}_{22}$ & $O^{-}+O_{2}=>O+O_{2}+e$ & Recombination & $6.9 \times 10^{-16}$ & {$[28]$} \\
$\mathrm{R}_{23}$ & $O^{-}+O_{3}+e=>O_{2}+O_{2}$ & Recombination & $1.02 \times 10^{-16}$ & {$[28]$} \\
$\mathrm{R}_{24}$ & $O_{2}^{-}+O=>O_{3}+e$ & Recombination & $1.5 \times 10^{-16}$ & {$[28]$} \\
$\mathrm{R}_{\mathbf{2 5}}$ & $O_{2}+O_{2}+O=>O_{3}+O_{2}$ & Association & $6 \times 10^{-46}$ & {$[28]$} \\
$\mathrm{R}_{26}$ & $O^{-}+O=>O_{2}+e$ & Recombination & $f(E / N)$ & {$[28]$} \\
$\mathrm{R}_{27}$ & $O_{2}+O_{2}=>O_{3}+O$ & Association & $2.95 \times 10^{-27}$ & {$[28]$} \\
$\mathrm{R}_{\mathbf{2 8}}$ & $O_{2}^{-}+O=>O_{2}+O^{-}$ & Ionic exchange & $1.5 \times 10^{-16}$ & {$[28]$} \\
$\mathrm{R}_{\mathbf{2 9}}$ & $O^{-}+O_{3}=>O_{2}^{-}+O$ & Ionic exchange & $3.01 \times 10^{-16}$ & {$[28]$} \\
\hline
\end{tabular}

\section{Results and discussion}

The electrical discharge in the oxygen gas (which is considered an electronegative gas in the electric discharge system) consists of a cylinder and a wire. We represent the two parts of the system in the concentric electrode system in the simulation model. Usually, the electric field is found in a concentric wire-cylinder system whose radii are a, which represents the radius of the inner electrode, and $b$ which represents the radius of the outer cylinder, i.e. the outer electrode. The electric field is not uniform, while the electric field strength between the electric discharge electrodes can be given as [6].

$$
E_{\text {radial }}=\frac{V_{\text {applied }}}{b \ln \frac{a}{b}}
$$

where $E_{\text {radial }}$ and $V_{\text {applied }}$ represent the radial electric field and the voltage applied across the discharge electrodes, respectively. The relation between the electric potential and the time is plotted in Figure 1.

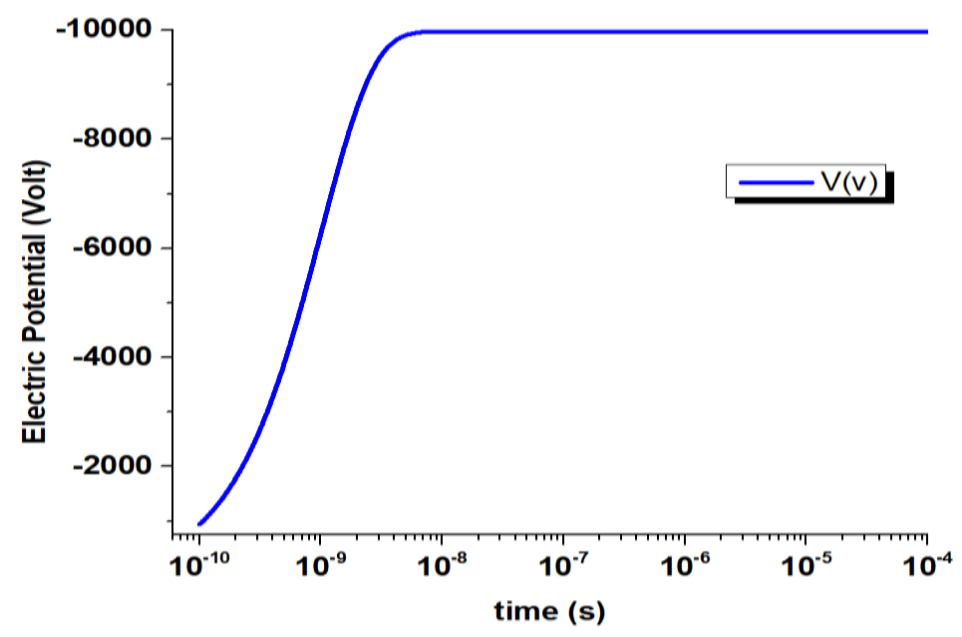

Figure 1- electrical potential on electrode

The radius of curvature of the inner electrode is less than the radius of curvature of the outer electrode, so the expectation is that the electrical breakdown occurs along the inner electrode. The highest electric field. The highest electric field $E_{b k}$ is related to the electric breakdown voltage $V_{b k}$ by the following expression [6].

$$
E_{b k}=\frac{V_{b k}}{b \ln \frac{a}{b}}
$$

The acceleration of the elementary electrons acquired from the field causes many collisions with the gas atoms. The ionization process occurs with the secondary electrons that have ions colliding (impinging) with the cathode. This process plays a crucial role in making the plasma self-sustained. It is reasonable to say that when the ions reach the cathode, they will gather near it, causing an increase in the electric field. This will be strong enough to push the electrons away from the cathode and accelerate them. These electrons face many collisions with the neutral atoms. In addition to the number of ions previously present in the 
very narrow ionization layer, they reach a specific value for the ionization process to occur according to equation (15). As we know, when the ions reach the cathode in large numbers, the cathode current begins to decrease so that the loss rate of positive ions becomes greater than their production rate. Figure 2 shows the relationship between the electric potential and the distance between the electrodes of the discharge at different diameters of the inner electrode in the negative corona.

On the other hand, the voltage of the separation between the discharge electrodes against a radii distance at different diameters of the inner electrode are plotted in Figure 3 .The decrease in the voltage with the increase in radii values is regular near the inner electrode. The positive ions are focused after the electrons are pushed from the same electrode. Therefore, it is evident. Therefore, it is evident that the concentration of electrons is high near the grounded electrode. Thus, the electric voltage decreases near the grounded electrode despite the attachment process that occurs with the gas by virtue of electronegative gas and the ionelectron recombination to form neutral atoms. The mechanisms of secondary electrons production ensures that the grounded electrode voltage is low [11]. The distribution of electron due to electrons departing from the cathode and the electron density near the sheath is roughly zero [29]. The spatial distribution of the electrons is shown in Figure 3. The electric potential increases steadily with the increase in the inner electrode diameter, whereas the rate of the electron path increases with increasing temperature [11]. This increase gives an additional opportunity for ions to collide with the electrode, thus leading to an increase in the total number of electrons.

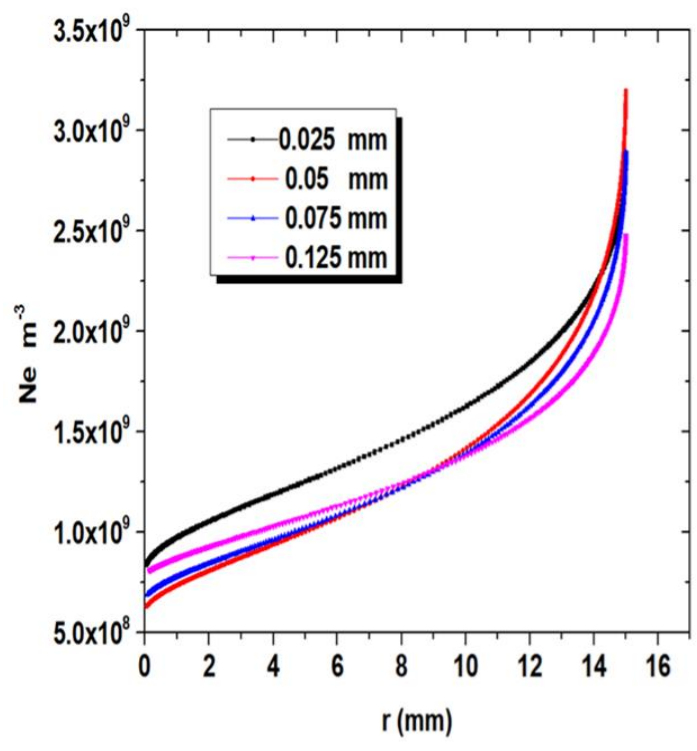

Figure-2 The relation between the electric potential and the radial distance as a function of the variable radii of the inner electrode.

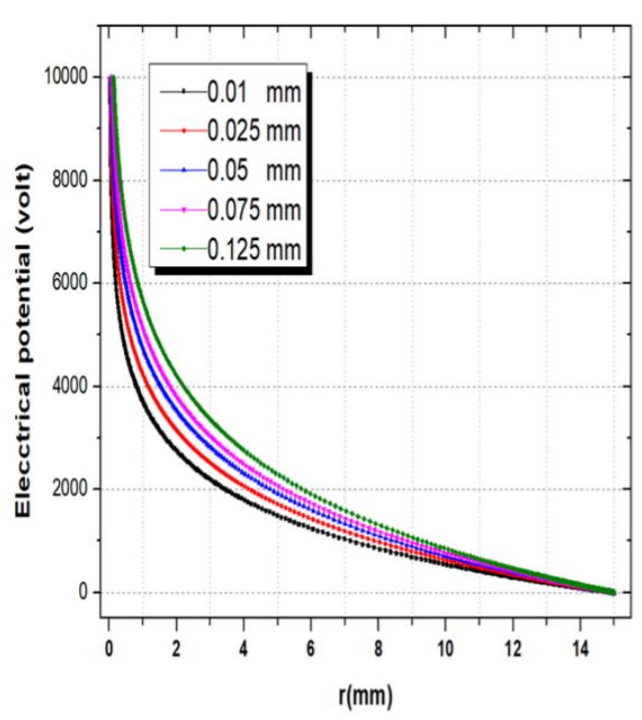

Figure-3 The spatial distribution of the electrons with radial distance as a function of the variable radii of the inner electrode.

The spatial distribution of the electrons in the inter-electrode distance provides a clear indication that the strong repulsion of the electrons by the cathode leads to a decrease in their density near the cathode. However, they begin to increase steadily through the taken path until reaching the grounded electrode. Because of the applied voltage remaining constant, there is no additional acceleration to create more collisions to raise the electron temperature by the energy gained from the field. Therefore, increasing the radius does not change the electron density.

The spatial distribution of the positive ions as a function of the inner electrode diameter, represented in corona electrode, is shown in Figure 4. In contrast to what happened with electrons, which were few at the surface of the negative wires, the positive ions decreased with increasing the distance and moved away from the surface of the wire then their the 
number of ions is decreasing. Therefore, the positive ions carry most of the overall current at the surface. The ion density decreases spatially and harmoniously with the distance between the electrodes, reaching zero outside the boundary of the plasma $[11,30]$. In brief, the decrease in the density of the positive ions with the increase in the electrode diameter indicates an increase in the field. The inset in Figure 4 provides a summary of the change in the positive ion density against wire diameter.

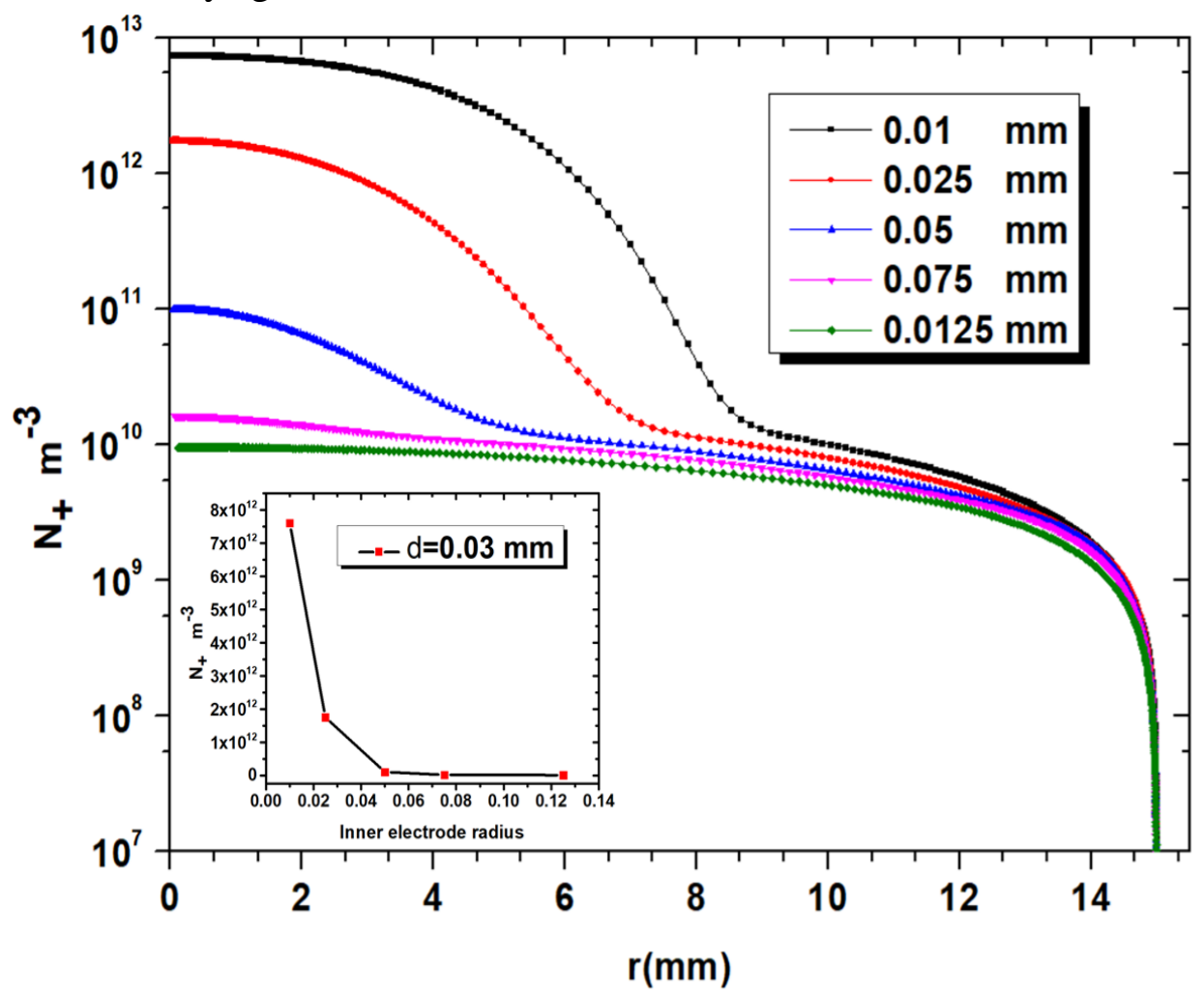

Figure 4-The spatial distribution of positive ions with the radial distance as a function of different inner electrode diameter values.

It is clear for all the diameters used for the inner diameter that there is a systematic decrease with increasing time so that the electron density is very low near the negative electrode

. In contrast, the positive ions gather near the negative corona electrode to reach the large density. This behavior gives an explanation about how the electric field accelerates the electrons when electrons cross the space between the electrodes. This space can be divided into several zones [11]. In these zones, the electrons are of varying distribution down to the grounded electrode, so the number of electrons is too low by the losses mechanisms mentioned above. 


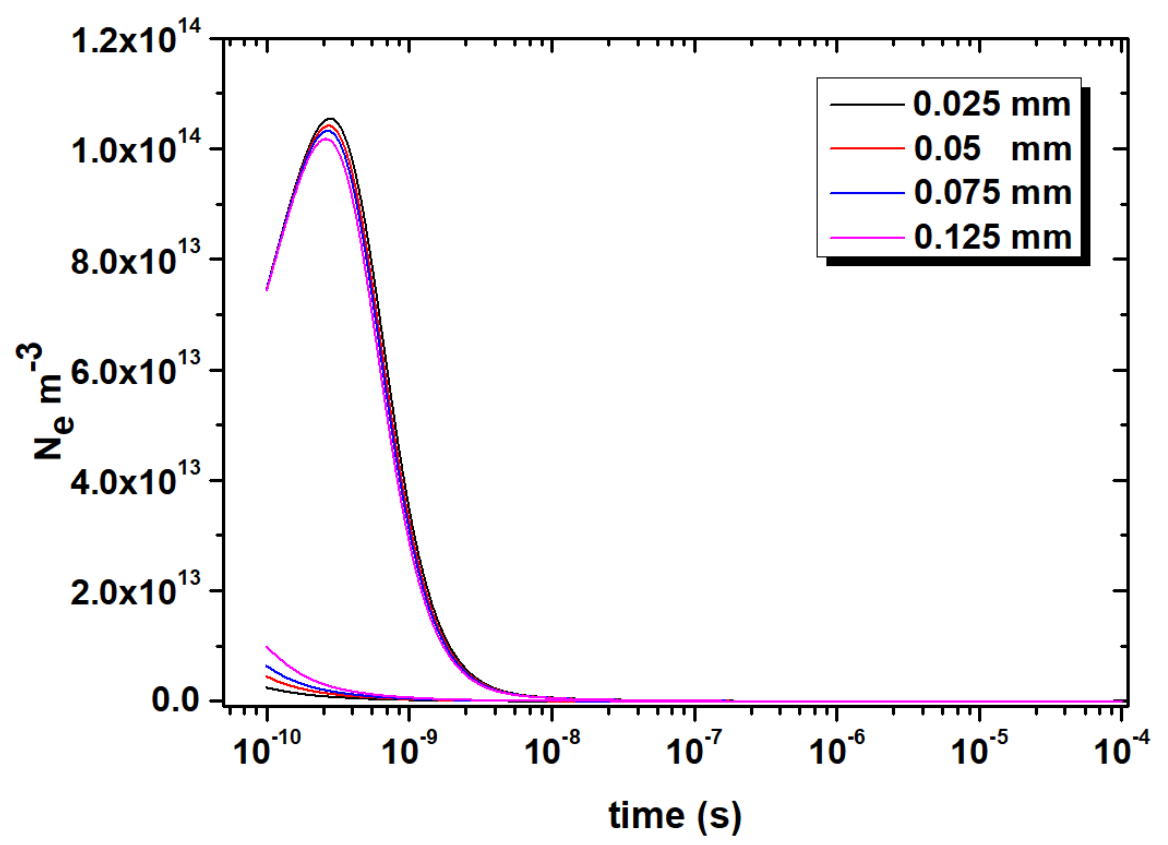

Figure 5- The distribution of electron density with the time along the radial distance as a function of inner electrode diameters.

The applied negative voltage drives the negative ions. This action is caused by the repulsion between the negative ions and the cathode, thus they are drifted away from the cathode. Therefore, the density is low in the cathode sheath region. At distances of $9,11,12$, 12.5 , and $14.1 \mathrm{~mm}$ for $0.01,0.025,0.05,0.075$ and $0.125 \mathrm{~mm}$ diameters, respectively, the changes in the distribution of the negative ions are evident, as it shown in Figure 6. A significant increase in the density of negative ions is obvious when entering the diffusion region. It is beneficial here to mention that the negative ions are heavy, so their mobility is slow. This makes the density of negative ions higher at the diffusion region. The electrical discharge of the negative corona, when increasing the diameter of the wire, creates an increase in the density of the electrons. However, the expansion of the plasma itself, along with the increase in the electrode diameter, leads to an increase in the energy of the electrons (the electrons near the surface of the wire are more energetic compared to the electrons at the edges). Then, a state of increased interactions will be reached. These interactions include several processes inside the plasma. Thus, the recombination process increases to form the neutral atom and the diffusion. This mechanism will lead to decrease electron density, while the density of negative ions also decreases. Additionally, the negative ions density decreases with increasing wire diameter $[9,11,17]$. 


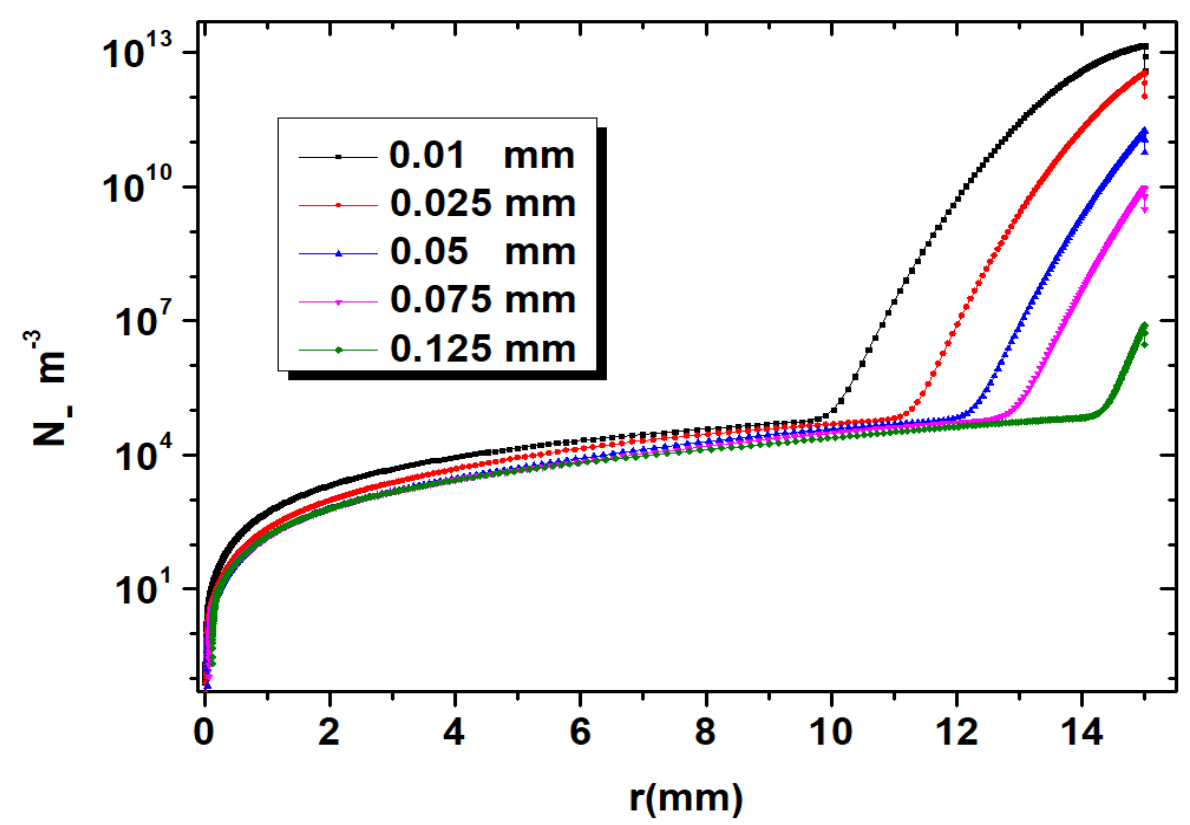

Figure 6-The distribution of negative ion density with the radial distance as a function of different inner electrode diameter values.

The spatial distribution of ozone density at different inner electrode diameter values is revealed in Figure 7. The ionization region is a shell surrounding the negative electrode, i.e. around the surface area of the wire with a certain small distance. Outside the boundaries of ionization, there is a balance (quality principle) between the process of producing positive ions and the process of attachment to form negative ions in electronegative gases [17]. Ozone is generated in the high-voltage area and appears at a short distance from the negative electrode of the corona discharge. At high voltage, the electron acquires high energy from the field, which is sufficient to complete the process of oxygen dissociation. Subsequently, this atom interacts with the oxygen molecule to generate ozone. The dissociation occurs at the electrode. The local field plays a major role in ozone generation that occurs near the electrode of the negative corona. The internal local field is very high and close to the external field. However, the external field dose not dominate in ozone generation, compared to the internal local field. The inset in Figure 7 represents the highest values of ozone production at all of the negative electrode diameters of the negative corona discharge. The greater diameter of the electrode is, the greater surface area of the wire it becomes, as mentioned above. This expansion of the plasma causes an expansion of the ionization region; therefore, the probability of ionization and the production of positive and negative ions surpasses the process of ozone production. In case of the small radius, plasma expansion is reduced and the zone of ozone generation becomes closer to the negative corona electrode. The ozone generation becomes greater when the negative corona electrode diameter is increased [14]. 


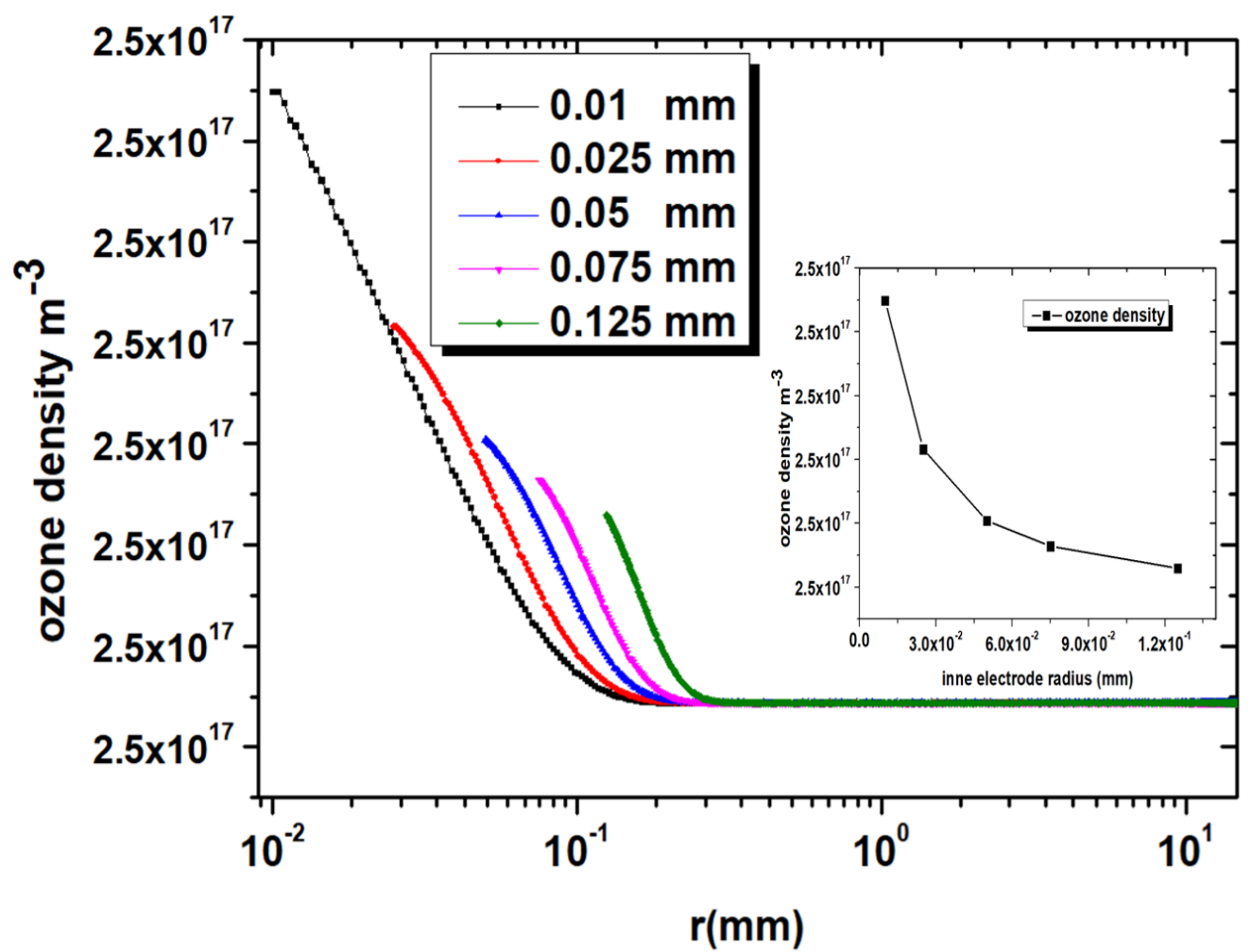

Figure 7- The spatial distribution of ozone density.

\section{Conclusions}

We introduced a numerical modeling in one dimension, simulated by using COMSOL Multiphysics software to study the role of the inner electrode radius on the oxygen negative corona discharge in coaxial electrodes geometry. The results reveal that the DC negative corona electrical discharge at atmospheric pressure exhibited a decrease in the electrical voltage when moving away from the cathode and then approaching the grounded electrode. This decrease behavior was found to be similarly for all studied diameters. Moreover, the result showed that the distribution of the electrons becomes larger near the ground electrode due to their repulsion from the cathode. The distribution of the positive ions reaches high values near the negative corona electrode, with the layer of ions being as a sheath adjacent to the surface of the wire. There was a harmonious increase in the distribution of negative ions and the distance between the electrodes. The surface area of the smaller electrode showed highest values of ozone density production. Finally, the results given by the proposed model are fairly consistent with those of previous studies in the field.

\section{Acknowledgement}

I would like to express my sincere thanks to the University of Mosul, College of Education for Pure Sciences and Physics Department for their unwavering support.

\section{References}

[1] A. Ala'F and A. Hasaani, "Experimental Study of Pulsed Electrical Discharge in CylindricallyTipped of Plasma Switch," Iraqi Journal of Science, vol. 58, pp. 951-960, 2017.

[2] K. Adamiak and P. Atten, "Simulation of corona discharge in point-plane configuration," Journal of electrostatics, vol. 61, pp. 85-98, 2004.

[3] M. Abdel-Salam and Z. Al-Hamouz, "Novel finite-element analysis of space-charge modified fields," IEE Proceedings-Science, Measurement and Technology, vol. 141, pp. 369-378, 1994.

[4] J. Cross and J. Beattie, "Positive glow corona in quasi-uniform fields," Canadian Electrical Engineering Journal, vol. 5, pp. 22-32, 1980. 
[5] M. Khalifa and M. Abdel-Salam, "Calculating the surface fields of conductors in corona," in Proceedings of the Institution of Electrical Engineers, 1973, pp. 1574-1575.

[6] L. K. Kenneth, Electrostatic Discharge. New York. Taylor \& Francis Group, LLC. CRC Press, 2006.

[7] J.-S. Chang, P. A. Lawless, and T. Yamamoto, "Corona discharge processes," IEEE Transactions on plasma science, vol. 19, pp. 1152-1166, 1991.

[8] J. Kuffel and P. Kuffel, High voltage engineering fundamentals: Elsevier, 2000.

[9] J. Chen and J. H. Davidson, "Electron density and energy distributions in the positive DC corona: interpretation for corona-enhanced chemical reactions," Plasma Chemistry and Plasma Processing, vol. 22, pp. 199-224, 2002.

[10] C. Zogning, J. Lobry, and F. Moiny, "Comparison between Positive and Negative Corona Discharges by Hydrodynamic Plasma Simulations," in 2020 International Symposium on Electrical Insulating Materials (ISEIM), 2020, pp. 552-560.

[11] J. Chen and J. H. Davidson, "Model of the negative DC corona plasma: Comparison to the positive DC corona plasma," Plasma chemistry and plasma processing, vol. 23, pp. 83-102, 2003.

[12] Y. Zheng, B. Zhang, and J. He, "Current-voltage characteristics of dc corona discharges in air between coaxial cylinders," Physics of Plasmas, vol. 22, p. 023501, 2015.

[13] M. K. Jassim, E. A. Jawad, and J. K. Alsaide, "Effect of Temperature on the Working Parameters of Negative Corona Discharge with Coaxial Electrodes Configuration," Iraqi Journal of Science, pp. 1977-1984, 2019.

[14] K. Yanallah, F. Pontiga, A. Fernandez-Rueda, A. Castellanos, and A. Belasri, "Ozone generation by negative corona discharge: the effect of Joule heating," Journal of Physics D: Applied Physics, vol. 41, p. 195206, 2008.

[15] A. Yehia and A. Mizuno, "Ozone generation by negative direct current corona discharges in dry air fed coaxial wire-cylinder reactors," Journal of applied physics, vol. 113, p. 183301, 2013.

[16] K. A. Aadim, "Simulation and experimental study of Pin-Plate DC discharge plasma technique," Iraqi Journal of Physics (IJP), vol. 15, pp. 138-147, 2017.

[17] J. Chen and J. H. Davidson, "Ozone production in the negative DC corona: The dependence of discharge polarity," Plasma chemistry and plasma processing, vol. 23, pp. 501-518, 2003.

[18] H.-j. Wang, L.-x. Sheng, and D.-x. Xu, "Effects of temperature and discharge parameters on ozone concentration of negative corona discharge," Journal of Environmental Sciences, vol. 15, pp. 796-799, 2003.

[19] S. Pekárek, "Effect of polarity on ozone production of DC corona discharge with and without photocatalyst," in 19th International Symposium on Plasma Chemistry, Bochum, 2009.

[20] F. d. P. Pontiga Romero, A. Fernández Rueda, H. Moreno González, and A. Castellanos Mata, "Negative corona discharge in synthetic and dry air," in 19th Europhysics Conference on the Atomic and Molecular Physics of Ionized Gases (2008), 2008.

[21] G. Karman, "Simulation and analysis of corona currents in large scale coaxial geometry due to triangular voltages," Diploma work in the Master Program electric power engineering, 2013.

[22] A. M. Howatson, An introduction to gas discharges: Pergamon international library of science, technology, engineering and social studies: Elsevier, 2013.

[23] N.-K. Kim, S.-H. Lee, G. E. Georghiou, D.-W. Kim, and D.-H. Kim, "Accurate prediction method of breakdown voltage in air at atmospheric pressure," Journal of Electrical Engineering and Technology, vol. 7, pp. 97-102, 2012.

[24] D. N. Saleh, Q. T. Algwari, and F. K. Amoori, "Numerical Simulation of the Trichel-Pulse in SF 6 at Atmospheric Pressure," IEEE Transactions on Plasma Science, vol. 47, pp. 427-433, 2018.

[25] A. Pateau, A. Rhallabi, M.-C. Fernandez, M. Boufnichel, and F. Roqueta, "Modeling of inductively coupled plasma SF6/O2/Ar plasma discharge: Effect of $\mathrm{O} 2$ on the plasma kinetic properties," Journal of Vacuum Science \& Technology A: Vacuum, Surfaces, and Films, vol. 32, p. 021303, 2014.

[26] G. Hagelaar and L. Pitchford, " Solving the Boltzmann equation to obtain electron transport coefficients and rate coefficients for fluid models," Plasma Sources Sci. Technol. vol. 14, pp. $722,2005$. 
[27] K.-L. Liu, R.-J. Liao, and X.-T. Zhao, "Numerical simulation of the characteristics of electrons in bar-plate DC negative corona discharge based on a plasma chemical model," Journal of Electrical Engineering and Technology, vol. 10, pp. 1804-1814, 2015.

[28] LXCat: Accessed 1 Nov 2017 electron scattering database, University of Toulouse, France. ww.lxcat.net.

[29] X. Liu, R. Xian, P. Yu, Y. Pei, X. Lv, X. Sun, et al., "Numerical simulation transient electron distribution of direct current negative corona discharge in air," in IOP Conference Series: Earth and Environmental Science, 2017, p. 012102.

[30] A. Sparavigna and R. Wolf, "Electron and ion densities in corona plasma," Czechoslovak Journal of Physics, vol. 56, pp. B1062-B1067, 2006. 